\title{
Sistematización de experiencias "Proyecto Formación de Formadores", Cultura Corazón Adentro, Distrito Capital, Municipio Libertador, Venezuela (2013-2015)
}

\section{Resumen}

El presente artículo, tiene como objetivo socializar la experiencia desarrollada por el proyecto "Formación de Formadores" del Distrito Capital, Municipio Libertador durante el período abril 2013 a marzo 2015. Este proyecto fue coordinado entre el Centro Nacional de Superación para la Cultura del Ministerio de Cultura de Cuba y la Dirección del Gabinete Cultural del distrito capital de Caracas, del Ministerio del Poder Popular para la Cultura de Venezuela, para diagnosticar las necesidades de capacitación de los activadores y gestores culturales que desarrollan su labor en las comunidades y para establecer estrategias de capacitación que diesen respuesta a estas necesidades y las de cuantos actores sociales participan en el desarrollo cultural del distrito capital. Representa, la expresión del esfuerzo realizado por un equipo de 14 docentes-investigadores cubanos en territorio venezolano, en apoyo a las principales políticas y estrategias de trabajo cultural del Ministerio de Cultura venezolano. Expresa, los principales resultados de un proceso docente concreto, desarrollado sobre

1 Doctor en Ciencias Jurídicas (PhD), Máster en Globalización y Derecho. Máster en Asesoría Jurídica. DocenteInvestigador de la Universidad Metropolitana (UMET), Quito, Ecuador. Docente de la Cátedra UNESCO "Cultura y Desarrollo" del Centro Nacional de Superación para la Cultura de Cuba e Investigador adjunto del Instituto de Investigación y Desarrollo de la Cultura Cubana "Juan Marinello" 
los principios de la Educación Popular y la Sistematización de Experiencias, como metodología de investigación acción, participación.

Palabras clave: Sistematización de experiencias, Educación Popular, Trabajo Comunitario, Formación de Formadores.

\begin{abstract}
The purpose of this article is to socialize the experience developed by the "Formation of Instructors" project of the Distrito Capital, Libertador Municipality, Caracas, during the period April 2013 to March 2015. This project was coordinated between the National Upgrading Center for Culture of the Ministry of Culture from Cuba and the Cultural Cabinet of the capital district of Caracas, Ministry of Popular Power for Culture of Venezuela, to diagnose the training needs of the cultural activators and managers who develop their work in the communities and to establish strategies for training that respond to these needs and those of how many social actors participate in the cultural development of the capital district. It represents the expression of the effort made by a team of 14 Cuban teacher-researchers in Venezuelan territory, in support of the main cultural work policies and strategies of the Venezuelan Ministry of Culture. Expresses the main results of a specific teaching process, developed on the principles of Popular Education and the Systematization of Experiences, as a methodology of action research, participation.
\end{abstract}

Keywords: Systematization of experiences, Popular Education, Community Work, Formation of Educators. 


\section{Introducción}

Como proceso inherente a toda práctica que desea recuperar de manera descriptiva y analítica, el presente artículo trata de socializar los resultados del informe de sistematización de la experiencia de dos años (abril de 2013 a marzo de 2015) del proyecto "Formación de Formadores" desarrollado en el distrito capital, Municipio Libertador, Caracas, Venezuela por un grupo de gestores culturales cubanos, que con el trabajo conjunto de diferentes especialistas e instituciones culturales venezolanas apoyaron al desarrollo de las estrategias de desarrollo cultural del Ministerio del Poder Popular para la Cultura en Venezuela. Este grupo de gestores cubanos estuvo conformado por los Master en Ciencias: Mercedes Pico Cruzata, Mileidis Pi Moreno, Xiomara Bueno Rosales, Madelyn Torres, Emma Lorenzo Fernández, Alian Cárdenas González y Adalberto Guevara; los Licenciados: Maribel Diñeiro Proenza, Antonio Verdecia Marín, Roberto Machado Izaguirre, Duniesky Contreras Madrigal, Violexi Montejo Ocana, Jenicet Pupo de La Paz; y el Doctor en Ciencias Jurídicas (PhD) Oscar Alberto Pérez Peña, Coordinador del equipo.

La sistematización de experiencias es una metodología de investigación participativa (Lewin, 1992) iniciada por colectivos comprometidos con la Educación Popular en América Latina. La sistematización de experiencias, para algunos autores, como Cáceres y Ayllón, citados por Oscar Jara, nace relacionada al campo del Trabajo Social, específicamente con la profesionalización del servicio social de influencia norteamericana (Jara, 2001). Luego se desarrolla en los nace en los años 70 en un contexto de crisis socioeconómica en la mayoría de los países de la región y en el que el paradigma de la educación para el desarrollo desde la perspectiva de la teoría del capital humano estaba demostrando sus carencias. Surge de corrientes renovadoras de pensamiento ante la visión positivista y radical de la ciencia: el trabajo social reconceptualizado; la Educación de Adultos; la Educación Popular; la Teología de la Liberación; La Teoría de la Dependencia y la Investigación Acción Participativa. Todas ellas con su propio método y ejes de acción pero que confluyen en la construcción, reconstrucción y transformación de procesos con un sujeto para el bien de los otros (Gutierrez Rico, 2008).

La Sistematización de Experiencias, como ejercicio de producción de conocimiento crítico desde la práctica, ha ido adquiriendo más y más relevancia en las experiencias de educación popular de América Latina y también en otros contextos. Muchas veces confundida con la mera recopilación de datos o con la narración de eventos, o aún con la producción de un informe-síntesis de una experiencia, las conceptualizaciones en torno a la sistematización de las experiencias han ido generando interesantes puntos de reflexión en torno a su identidad específica.

Para Oscar Jara "La sistematización es aquella interpretación crítica de una o varias experiencias, que, a partir de su ordenamiento y reconstrucción, descubre o explicita la lógica del proceso vivido, los factores que han intervenido en dicho proceso, cómo se han relacionado entre sí, y por qué lo han hecho de ese modo" (Jara Holliday, 2018)

Sistematizar es en esencia recuperar, ordenar, precisar y clarificar con carácter científico el saber que se obtiene del servicio ofrecido o alguna práctica profesional protagonizada en particular, constituyendo una oportunidad de reflexión analítica para interpretar lo sucedido, comprenderlo y 
obtener un producto consistente y sustentado, a partir del cual es posible transmitir la experiencia, confrontarla con otras y con el conocimiento teórico existente, y así contribuir a una acumulación de conocimientos generados desde y para la práctica.

El desarrollo de esta labor, en el período referido de actuación -además de reconocer, clasificar e interpretar los procesos inherentes al proyecto Formación de Formadores del distrito capital, Municipio Libertador-, supone la reflexión colectiva para la construcción de instrumentos y herramientas surgidas desde el análisis agudo de sus prácticas, desempeño organizativo y académico, en particular.

El trabajo fue desarrollado siguiendo los siguientes objetivos

\section{Objetivo general}

1. Sistematizar la experiencia desarrollada por el proyecto "Formación de Formadores" del Distrito Capital, Municipio Libertador durante el período abril 2013 a marzo 2015 posibilitando el análisis crítico del proceso.

\section{Objetivos específicos:}

1. Recuperar la memoria histórica de la experiencia sistematizada por el proyecto "Formación de Formadores" del Distrito Capital, Municipio Libertador durante el período abril 2013 a marzo del 2015.

2. Identificar, los resultados de la experiencia en cada área de acción realizando un análisis crítico.

3. Elaborar el informe del proceso de sistematización a partir de las conclusiones, recomendaciones y socialización realizada en las diferentes etapas.

Para la conformación del grupo de sistematización se tuvo en cuenta la presencia de cada miembro del equipo de profesores del Proyecto "Formación de Formadores" del Distrito Capital, Municipio Libertador, Venezuela, con roles o tareas claramente definidas.

La participación de otros colaboradores, directivos o actores de la misión se garantizó a partir del análisis previo de la pertinencia y aportes que desde su perfil y/o funciones de trabajo, pudiesen realizar al análisis crítico de las experiencias a sistematizar.

\section{Materiales y métodos.}

En cuanto a la metodología, se ha tomado en cuenta la propuesta de Oscar Holliday Jara en sus diferentes textos derivados de su experiencia de más de treinta años, particularmente las referidas en su texto, Para sistematizar experiencias: una propuesta teórica y práctica de 1994 y que ha mantenido actualmente (Holliday Jara, 2018). Se ha tenido en cuenta tres momentos claves, condensando la metodología de Jara, que cuenta con 5 momentos o tiempos; resultando en este 
caso los siguientes:

- Recuperación del proceso.

Descripción de la experiencia: dónde y cuándo se realizó, qué actores participaron, con qué objetivos, resultados obtenidos. Se realizó una mirada ordenada de la práctica, en este caso con los registros de las experiencias en que se participó.

Recuperación del desarrollo del proceso: lo más completa posible, dando cuenta de todo lo sucedido en este caso lo programado, considerando los puntos de vista de los diversos participantes. Se ha tenido en cuenta acá el objetivo y objeto de la sistematización, así como los ejes de sistematización

- Análisis e interpretación del proceso

El objetivo es explicar los procesos vividos, sacando a la luz los nuevos conocimientos obtenidos durante las experiencias. Se realizó respondiendo a las preguntas clave planteadas en los momentos del análisis, considerando y relacionando toda la información. El momento de análisis e interpretación culminó con el ordenamiento de las lecciones que la experiencia y su sistematización han permitido alcanzar, así como el análisis de propuestas para mejorar los modos de actuación. Se trata de una nueva y diferente mirada a la experiencia: la del conocimiento producido, que se traduce generalmente en recomendaciones para mejorar nuestras prácticas personales y profesionales. Se ha reconstruido la historia y se clasificado toda la información para ser procesada y analizada. Del mismo modo, se ha analizado e interpretado el proceso de forma crítica.

- Socialización,

El último momento del proceso es la socialización de los resultados de la sistematización, el cual se ha ido desarrollando de forma sistemática entre los propios integrantes del equipo para luego consolidar los principales aprendizajes. En esta etapa se formularon las conclusiones y recomendaciones para luego comunicar los aprendizajes.

Las técnicas fundamentales para la búsqueda y procesamiento de información fueron:

Observación: El acercamiento a la realidad no es posible sin una mirada aguda, detallada y adecuada a los propósitos deseados para la descripción. De modo que la asistencia y participación en los diferentes espacios permite la visualización real de las cotidianidades y realidades internas y externas del proceso. En este sentido, los actores/observadores han problematizado su realidad a través del diálogo con otros actores de la experiencia, reflexionando sobre la interpretación y construcción de la labor que se realizaba. Se ha dialogado sobre las observaciones realizadas por los observadores y los objetos de conocimiento (Cendales, G. y Torres Alfonso, 2003)

Análisis documental: Fuentes teóricas y metodológicas contenidas en la bibliografía especializada sobre el tema y en los diferentes documentos relacionados con el proceso (Barbosa Chacón, 
Barbosa Herrera y Rodríguez Villabona, 2013); diario de campo que permitió el registro sistemático, permanente y organizado de las actividades observadas y las informaciones recogidas; memoria audiovisual y fotográfica del proceso (Middleton y Edwards, 1992)

La entrevista a actores de la experiencia ha permitido el registro y análisis de los estados de opinión, así como las expectativas sobre los procesos en los cuales formaron parte (Sanin Betancourt, 2016)

Son importantes los resultados del empleo de la capacitación en los procesos de sistematización de experiencias (Rodríguez Sosa, 2019). Se han realizado evaluaciones docentes de forma colectiva, así como individual por la coordinación del equipo de profesores, de forma tal que sirvieran de presupuestos teóricos y metodológicos para corroborar la pertinencia del proceso formación de formadores. En ese sentido, se han tenido en cuenta las acciones docentes impartidas por los diferentes especialistas de los procesos esenciales de la misión.

\section{Resultados y discusión}

\section{Proceso de reconstrucción. Interpretación crítica de las experiencias. Actividades y procesos académicos del proyecto "Formación de Formadores" del Distrito Capital, Municipio Libertador.}

Elaboración y actualización del diagnóstico de Necesidades de Capacitación del Distrito Capital. Municipio Libertador. (2013-2015).

El informe de diagnóstico, en su etapa inicial de diseño, tuvo como fecha de realización del 4 al 8 de junio de 2013. Como paso previo al diseño e implementación de las acciones de formación y desarrollo de competencias del talento humano venezolano implicado en las estrategias de desarrollo cultural en el municipio Libertador. Los procesos de actualización del mismo se han realizado de manera sistemática por cortes trimestrales y presentación anual de un informe. La actualización culminó en fecha 5 de marzo del 2015.

Es válido destacar además que los escenarios para la aplicación de las técnicas de investigación, la selección de las muestras y universo se plantean en correspondencia con las políticas de Gobierno de la Revolución Bolivariana.

\section{Etapa Inicial (mayo 2013 - marzo 2014).}

Inicialmente el trabajo del Proceso "Formación de formadores" centró como muestra principal a los animadores y animadoras venezolanos de la Misión Cultura "Corazón Adentro" emplazados en las diferentes comunidades de las parroquias del municipio Libertador. Ello significa que los primeros acercamientos a las necesidades de capacitación declaradas evidenciaron en primera instancia elementos asociados al perfeccionamiento técnico-metodológico en las diferentes manifestaciones y en menor medida temas relacionados con la gestión integral de los procesos culturales y los 
procesos asociados al trabajo comunitario de manera integral.

Fueron identificadas inicialmente un total de 75 Necesidades de Capacitación en 11 líneas temáticas

- Político- ideológico, valores y estética revolucionaria,

- Gestión Integral de los procesos culturales,

- Dirección,

- Trabajo comunitario,

- Identidad y patrimonio cultural y natural,

- Metodologías y didácticas aplicadas,

- Literatura, Artes Plásticas, Teatro, Música, Danza.

Luego del proceso de actualización del diagnóstico en esa etapa, sumaron un total de 83 el total de necesidades de capacitación identificadas. Las incorporadas:

- Precisiones y estrategias del trabajo comunitario para refugios, urbanizaciones, Comités de Cultura.

- Bases legales para la articulación del Poder Popular.

- Educación Popular.

- Andragogía.

- Derechos culturales.

- Didácticas de Museos.

- Arte latinoamericano contemporáneo.

- Sistema Económico Comunal

\section{Etapa abril de 2014 a marzo de 2015.}

Durante este período el proceso de actualización del diagnóstico se realizó teniendo en cuenta los contenidos temáticos y ejes conceptuales establecidos para la actividad docente de la Cátedra Permanente "Soberanía y Cultura Comunal", así como otros identificados por los profesores en las diferentes comunidades e instituciones culturales y afines. 
De conjunto con los facilitadores de la Misión Académica, la participación en este nuevo escenario permitió al proceso "Formación de Formadores" la revisión de los documentos normativos y metodológicos que reflejaban una actualización de las prioridades formativas del gabinete y la Fundación "Misión Cultura" en general.

A partir del mes de junio de 2014 y hasta marzo 2015, en correspondencia con el llamado hecho por el presidente de la República Bolivariana de Venezuela Nicolás Maduro a reducir los índices de pobreza extrema en el país, comienza el despliegue de la colaboración cubana hacia 26 comunidades identificadas de ese tipo en el municipio Libertador y desde los meses de agosto y septiembre a los urbanismos. También se han tenido en cuenta el personal y especialistas de las diferentes instituciones culturales del distrito y de otras que indirectamente contribuyen a las estrategias de desarrollo cultural.

Nuevos ejes temáticos planteados para la Cátedra Permanente

- Políticas culturales

- Diversidad cultural

- Animación integral de los procesos socioculturales

- Entre estos ejes se sumaron 23 nuevas necesidades.

Necesidades identificadas en zonas de extrema pobreza, urbanismos, comunidades e instituciones culturales y otras.

Se han identificado necesidades de formación de animadores (as) culturales y diferentes actores sociales que participan en el desarrollo cultural a partir del abordaje realizado a las comunidades del distrito (incluye las 26 zonas de extrema pobreza y urbanismos), instituciones culturales, miembros de las estructuras del Poder Popular en ellas y otras instituciones (Museos, Universidad Bolivariana, Defensoría del Pueblo, Instituto del Patrimonio Cultural) que desarrollan acciones afines a la cultura. Se suman entonces a las 23 anteriores, 12, para un total de 35 nuevas, en este caso:

- Diagnóstico participativo, con énfasis en zonas de extrema pobreza y urbanismos.

- Bases legales para la articulación del Poder Popular.

- Animación integral de los procesos culturales

- Referentes teóricos y prácticos sobre protección de bienes culturales y lucha contra el tráfico ilícito del Patrimonio Cultural.

- Planificación

- Planificación docente 
- Gestión por competencias

- Gestión de proyectos socioproductivos y socioculturales.

- Gestión de los procesos de dirección y planificación comunal.

- Prevención.

- Investigación acción participación.

- Formación de valores y conciencia revolucionaria.

Se cierra el diagnóstico con un total de 118 necesidades de capacitación identificadas.

\section{Acciones docentes desarrolladas en el período dirigidas a beneficiarios meta. Principales modalidades empleadas y resultados en el orden cualitativo y cuantitativo (2013-2014) y (2014 2015).}

\section{Acciones docentes realizadas (mayo 2013- marzo 2014):}

Las acciones académicas realizadas por el equipo de profesores han sido abordadas fundamentalmente desde el Taller, la Asesoría, las conferencias y los cursos como modalidades docentes, siempre bajo los presupuestos teóricos y metodológicos de la Educación Popular. Constituyen un total de 70 (1 Diagnóstico, 27 talleres, 1 Seminario, 12 Conferencias, 4 cursos, 25 Asesorías) para un total de 857 beneficiarios meta (453 animadores y animadoras culturales y 404 otros actores sociales). Lo que propició el desarrollo de competencias en beneficio del proceso de gestión cultural, la labor investigativa, así como la articulación de estrategias para el mejoramiento de la calidad de vida en la comunidad.

\section{Etapa abril de 2014 a marzo de 2015}

En este año las acciones académicas realizadas por el equipo de profesores han sido abordadas también desde el Taller, la Asesoría y las conferencias. Constituyen un total de 101 (1 Diagnóstico, 63 talleres, 26 Conferencias, 1 curso, 10 Asesorías) para un total de 1516 beneficiarios meta (312 animadores y animadoras culturales y 1204 actores sociales varios).

A modo de conclusión, entre los dos años de trabajo del equipo, han sido 2373 beneficiarios meta capacitados (765 animadores y animadoras culturales, 1608 otros actores sociales) y 170 acciones docentes

(1 diagnóstico, 90 talleres, 38 conferencias, 5 cursos, 35 asesorías y 1 seminario). Las modalidades fundamentales de la superación fueron los talleres, las asesorías y las conferencias 
y en menor medida los cursos. Esto último obedece, en nuestra opinión, a las dinámicas internas de los actores sociales capacitados que no se encuentran en un lugar específico para recibir dichas acciones, así como el hecho de que muchas de ellas se gestionaron por los profesores y no pudieron materializarse debido a la falta de organización de la parte venezolana que las solicitaba.

Los procesos académicos realizados por el equipo de profesores hasta la fecha han sido abordados desde la Educación Popular y el principio de "aprender haciendo". Ha jugado un papel importante dentro de las acciones realizadas, el fomento de la práctica investigativa como herramienta para la búsqueda de soluciones, la gestión y evaluación de los procesos organizativos en el trabajo comunitario. Los talleres de sistematización, las asesorías a trabajos científicos, las acciones de sensibilización para la construcción del diagnóstico sociocultural participativo en las comunidades y los procesos de acompañamiento y evaluación a los eventos de intercambio de saberes comunitarios.

Estrategia de formación orientada a zonas de extrema pobreza y nuevos urbanismos. Resultados.

Ciudad Caribia:

Como parte de las acciones docentes del equipo, a partir del mes de junio de 2013 comenzaron a desarrollarse acciones docentes en Ciudad Caribia en aras de fortalecer la articulación de los diferentes actores sociales de esta comunidad. En ese sentido, y partir de los diferentes talleres allí realizados se presentan como principales resultados de este trabajo:

- El Acercamiento y socialización a los actores locales y comunitarios.

- Se incorporaron nuevos actores estratégicos al Grupo Gestor.

- Se incrementó el grado de motivación de las instancias técnico- administrativas a partir de la entrada del proceso.

- Se aprecia un mayor grado de respaldo por el gobierno local.

- Se incrementaron el número de alianzas estratégicas.

- Aumentó el grado de participación de la comunidad en la toma de decisiones contribuyendo a generar una conciencia de accionar como sujetos de cambios.

- Crecimiento personal y grupal en los participantes.

- Mayor interés de la población por actividades socioculturales y por espacios de aprendizaje, lo que demuestra sentimiento de pertenencia al desarrollo endógeno.

- En algunos casos los gestores culturales y líderes naturales se han convertido en multiplicadores de las experiencias, adecuándolas a las circunstancias particulares de sus comunidades y grupos 
- El desarrollo de Talleres y encuentros con actores estratégicos, lo que ha permitido su articulación.

- Multiplicación y socialización de la propuesta, pues han comenzado a organizarse a este efecto para desarrollar la capacitación hacia las diferentes terrazas.

- Acciones de compromiso y acuerdos de trabajo.

- Se diseñó una Estrategia de Trabajo para el abordaje de las urbanizaciones del distrito capital y de otros estados de Venezuela que tiene como particularidad la utilización de la cultura como eje transformador sociocultural. Se valora la posibilidad de trabajo conjunto al respecto con la Fundación Oro Negro.

Zonas de extrema pobreza y urbanismos:

A partir del mes de julio de 2014 se redimensionaron las líneas estratégicas de la misión, por solicitudes de la máxima dirección del país, priorizando el trabajo en las comunidades de extrema pobreza y urbanizaciones en aras de contribuir a su transformación cultural. En ese sentido se diseñó un Estrategia de formación orientada a fortalecer las competencias profesionales de nuestros colaboradores cubanos para acometer la labor, así como al desarrollo de la transformación en estas comunidades, a modo de contribución a este proceso. La estrategia quedó conformada en dos etapas:

\section{Primera etapa (julio- octubre 2014)}

Para esta etapa se planteó como objetivo fundamental el desarrollo de acciones de atención metodológica y capacitación que contribuyeran al fortalecimiento de competencias profesionales de los instructores de arte.

Para ello se tuvo en cuenta la realización de

1. Acciones de coordinación conjunta con el equipo metodológico del estado para la determinación de necesidades de capacitación de los instructores de arte afectados para el trabajo en estas comunidades y en correspondencia con su nuevo perfil de actuación, promotor cultural.

2. Talleres de sensibilización y/o desarrollo de competencias en el marco de las preparaciones metodológicas que abordaron como temáticas el diagnóstico participativo comunitario, elementos para el diseño y facilitación de proyectos socioculturales, socioproductivos, los panes de acción cultural y principios del trabajo comunitario en general.

3. Acompañamiento in situ, -en las jornadas de trabajo comunitario, visitas casa a casa, domingo de misiones, etc.- al colaborador en los procesos de aplicación del diagnóstico, y procesamiento de la información. 
El trabajo en las Comunidades de Extrema Pobreza del equipo de profesores de conjunto con el equipo metodológico se abordó desde una visión científica que permitió la concepción de estrategias marcadas por su objetividad, novedad y pertinencia.

1. Diseño y aplicación de una encuesta (agosto 2014), a los 26 colaboradores con el objetivo de identificar las principales necesidades formativas de los instructores de arte de caras al trabajo realizado, así como analizar la información preliminar que sobre su trabajo en las comunidades nos pudiera arrojar: niveles de articulación con las estructuras del Poder Popular presentes en las comunidades, los principales problemas en las mismas, niveles de urgencia en su solución, y principales acciones realizadas en favor de su solución; los índices de participación comunitaria, la presencia de líderes naturales y el grado de protagonismo, la existencia de consejos comunales, comunas y mesas de cultura, etc.

2. Realización del Primer Taller de Intercambio de Experiencias entre colaboradores ubicados en las Comunidades de Extrema Pobreza (10 y 11 de septiembre del 2014).

3. Sistematización del evento (octubre 2014) y realización de un informe donde -a partir del análisis de las relatorías del trabajo en comisiones-, se resumieron los principales resultados del mismo y se elaboró un grupo de recomendaciones y conclusiones en función de la atención formativa, metodológica y/o administrativa del colaborador ubicado en estas comunidades. Esta sistematización permitió:

- Impulsar la presencia del equipo de formación de formadores en los espacios de preparación metodológica con temas afines al perfil de promotor cultural que asume el profesional de la cultura que labora en las comunidades de extrema pobreza.

- Fortalecer desde el nivel teórico y metodológico los procesos de inducción de los colaboradores que ingresan a la misión y el papel que en ese sentido juega el proceso "Formación de Formadores".

- Confeccionar una guía metodológica para la realización de los talleres de sensibilización sobre el diagnóstico participativo.

\section{Segunda etapa (octubre- diciembre 2014 a marzo 2015).}

Esta etapa -aunque se planteaba su proyección-, se profundizó a partir de la sistematización del primer Taller de intercambio de experiencias y tuvo como objetivo fundamental el desarrollo de procesos formativos en las comunidades que contribuyan al fortalecimiento de la labor que realizan los instructores en las comunidades y a la articulación de las estructuras del Poder Popular a partir de acciones de capacitación que en las modalidades de talleres y asesorías fortalezcan los procesos de diagnóstico participativo comunitario, la ejecución de planes de acción culturales, proyectos socioculturales y/o socioproductivos y que contribuyan en consecuencia, a la capacitación de los voceros culturales, a la conformación del Comité de Cultura y las Mesas Culturales. 
Se exigió la presencia de los colaboradores en calidad de facilitadores y para asegurar la continuidad del proceso formativo-, también se desarrollaron acciones dirigidas a las estructuras del Poder Popular (concejos comunales, mesas técnicas y vocerías) pues constituyen conjuntamente con los actores sociales de la comunidad, beneficiarios meta de las acciones académicas emprendidas.

Principales acciones realizadas:

1. Conformación de dúos de trabajo (profesores de formación de formadores) para el abordaje integral de las comunidades de extrema pobreza del Distrito con una programación de trabajo de una entrada semanal.

La decisión de una entrada semanal respondió a la necesidad de asegurar los resultados en otros ámbitos de actuación académica del proceso como la Cátedra Permanente y otros beneficiarios metas e instituciones.

Esta estrategia permitió la entrada de los dúos con acciones concretas con los concejos comunales en las siguientes comunidades: Loma Grande, Cruz Alta, El Plan, Río Cristal, El Carmen, La Acequia, El Matadero, Taparitas.

2. Realización -y socialización en la preparación metodológica estadal-, de una guía metodológica para la realización de talleres de sensibilización y otra sobre el diagnóstico participativo y el trabajo comunitario en general que le permitió al instructor continuar el trabajo iniciado con la asesoría nuestra. El equipo de formación lo conformaron 7 profesores a distribuir por 26 zonas, de ahí que la entrada nuestra fue de un lado para facilitar el proceso comunitario desarrollado por el instructor y el animador y de otro, para desarrollar acciones dirigidas a solventar las necesidades formativas que tuviesen las diferentes comunidades y las diferentes estructuras del Poder Popular.

3. Acciones realizadas de forma conjunta con el equipo metodológico del estado

- Determinación de necesidades de capacitación de los instructores de arte.

- Desarrollo de talleres de inducción a los nuevos colaboradores en temas como: diagnóstico participativo comunitario, elementos para el diseño y facilitación de proyectos socioculturales, socio-productivos, articulación con las estructuras del Poder Popular en Venezuela, planes de acción cultural y principios del trabajo comunitario en general.

- Diseño y elaboración de preparaciones metodológicas en los temas precedentes.

- Sistematización y análisis permanente de los resultados de trabajo de los instructores ubicados en las 26 zonas. Diseño y Realización de preparaciones metodológicas para elaborar los programas de formación y para fortalecer las competencias profesionales del equipo de profesores y el equipo metodológico en temas afines a este trabajo.

- Elaboración de encuesta e informe conclusivo con sistematización de resultados acerca del abordaje casa a casa a las bases de misiones por el sistema de intermisiones. 
- Devolución de resultados del Primer Taller de Intercambio de Experiencias entre colaboradores ubicados en las Comunidades de Extrema Pobreza.

4. Acciones realizadas en las comunidades y con otros actores sociales

- Acompañamiento in situ, -en las jornadas de trabajo comunitario, visitas casa a casa, domingo de misiones, etc.- al colaborador en los procesos de aplicación del diagnóstico, y procesamiento de la información.

- Conformación de dúos de trabajo (profesores de formación de formadores) para el abordaje integral de las comunidades de extrema pobreza del distrito con una programación de trabajo de una entrada semanal. (para asegurar los resultados en otros ámbitos de actuación académica del proceso como la Cátedra Permanente y otros beneficiarios metas e instituciones).

- Desarrollo de talleres de sensibilización y talleres de diagnóstico participativo en las comunidades dirigidos a los diferentes actores sociales de las mismas y a las estructuras del Poder Popular, con la presencia del instructor de arte, de forma que este recibe la preparación como entrenamiento in situ y la continua en su comunidad, una vez realizados los dos primeros talleres.

- Confección de dos guías metodológicas para la realización de los talleres de sensibilización en las comunidades y sobre diagnóstico participativo.

- Realización -y socialización en la preparación metodológica estadal-, de las guías para talleres de sensibilización y sobre diagnóstico participativo, que le permita al instructor continuar el trabajo iniciado con la asesoría.

- Desarrollo de talleres o asesorías en las comunidades, dirigidos a los diferentes actores sociales o a los miembros de las estructuras del Poder Popular (Comunas, Concejos Comunales, mesas técnicas y vocerías), a partir de las solicitudes realizadas al equipo en diversos temas.

- Realización de acciones de familiarización con este trabajo en el marco de las actividades de la Cátedra Permanente, de forma tal que los miembros de la misión académica se sumen a la actividad formativa en las comunidades de extrema pobreza.

- Sistematización de la experiencia de trabajo.

Como principales resultados de toda esta labor se aprecia:

1. Se ha identificado la incorporación de, al menos, 17 animadores culturales al trabajo en zonas de extrema pobreza y urbanismos.

2. Se ha fortalecido el trabajo de los instructores en las diferentes zonas respecto a la 
integración con las estructuras del Poder Popular y con el sistema de intermisiones en particular, deporte y misión médica en pos de la transformación sociocultural de las comunidades.

3. Alto reconocimiento social del colaborador y su trabajo, por parte de las comunidades.

4. Se ha elevado el índice de participación por segmentos poblacionales en las diferentes actividades comunitarias con especial atención en la tercera edad, adolescentes y jóvenes.

5. Se ha atendido a grupos de riesgo y desventaja social.

6. Mayor nivel de articulación y participación de actores, instituciones y organizaciones (consejos comunales, misiones sociales) en la consolidación del trabajo comunitario integrado y el desarrollo sociocultural.

7. Mayor grado de satisfacción de las necesidades declaradas y sentidas de la población beneficiaria. (validado a partir de estados de opinión).

8. Pertinencia de los proyectos socioproductivos en el desarrollo de procesos de activación popular y de desarrollo endógeno.

9. Niveles de reducción desde el trabajo sociocultural, de toda manifestación que vaya en detrimento de las prácticas de convivencia, las relaciones de género y el medioambiente. Esto ha sido intencionado tanto en las acciones formativas como en las diferentes actividades comunitarias y talleres.

10. Mayor grado de contribución desde los procesos de asesoramiento, al desarrollo ideológicocultural del animador venezolano u otros actores sociales que inciden en las acciones de desarrollo sociocultural en las comunidades.

11. Mayor nivel de incidencia desde los procesos de formación y capacitación, al perfeccionamiento de las prácticas de gestión integral de los procesos culturales.

12. Incidencia en la calidad técnico-artística y metodológica de los servicios culturales en beneficio de una sólida formación ético-estética y el fortalecimiento de valores identitarios.

13. Contribución a la formación de actores populares que tributen a la salvaguardia de la cultura popular y la memoria histórica.

14. Mayores niveles de promoción, desde la comunidad, de iniciativas, proyectos, programas, estrategias enfocadas en generar nuevos esquemas de recreación, que se contrapongan a la cultura del exceso, la destrucción y las irrespetuosas relaciones humanas que genera el capitalismo.

15. Mayor comprometimiento y asunción del arte y la cultura en general como mecanismo dinamizador y formador de una cultura de paz. 
Principales desafíos

1. Fortalecer el diseño e implementación del diagnóstico y el plan de acción cultural desde una concepción participativa en su integralidad, lo que de materializarse permitiría mejores resultados.

2. Fortalecer la presencia y el abordaje en las comunidades de los animadores culturales y de operadores, facilitadores, especialistas de las diferentes instituciones culturales y personal nacional, en pos del trabajo comunitario.

3. Continuar trabajando en el perfeccionamiento del perfil del colaborador y del animador cultural a partir de las demandas de las comunidades de extrema pobreza en las que está insertado.

4. Impulsar los procesos de articulación con Concejos Comunales y Comunas para la ayuda, por concepto de transportación (Rutas comunales, Carnet), de colaboradores que no se encuentran fijos en estas comunidades.

5. Aún son insuficientes los materiales de trabajo para el apoyo desde todas las manifestaciones.

Es válido destacar los aportes realizados por la Coordinación Nacional del proyecto en términos de revisión y aprobación de programas, entrega de bibliografía actualizada, así como observaciones y recomendaciones metodológicas en beneficio, vinculadas con estrategias formativas para una mejor calidad de los procesos de coordinación y ejecución de las acciones docentes que planificó el equipo.

\section{Diseño y participación en proyectos de formación nacionales.}

A partir de las potencialidades del equipo de profesores, algunos de ellos participaron en el diseño y conformación de un Programa Nacional para la formación de promotores culturales para el frente Francisco de Miranda, otros en una Estrategia de Trabajo para el abordaje de las urbanizaciones del distrito capital y de otros estados de Venezuela y un profesor nuestro en el diseño de una Estrategia Formativa Nacional en temas de Patrimonio cultural para el desarrollo de acciones docentes en esta área, que fue llevada a 14 estados de Venezuela y en la cual participó en 8. En virtud de esta última se desarrolló un taller de Gestión integral del Patrimonio Cultural en el IPC y uno sobre Protección Jurídica del Patrimonio Cultural (junio 2014) estas acciones fueron impartidas a diversos actores sociales que participan en la protección del patrimonio cultural, entre los que se encuentran las comunidades, los depositarios de estos bienes culturales, los miembros de las plataformas en los Gabinetes y las alcaldías y gobernaciones.

\section{Sobre la participación en actividades con el Gabinete de Cultura y otros actores sociales.}

Reuniones de trabajo con la dirección del Gabinete Cultural del Distrito para la coordinación y planificación de acciones. 
Al realizarse el diagnóstico de necesidades de capacitación inicial, se entrevistaron a animadores, coordinadores, cultores y directivos de la Fundación Misión Cultura, coordinadores, metodólogos y colaboradores de la Misión Cultura Corazón Adentro y otros actores del desarrollo cultural en el Distrito Capital. Este proceso se fue acrecentando en la medida que se ha desarrollado el trabajo del equipo de profesores y ha cambiado la dirección del Gabinete Cultural.

Es importante destacar que la presencia en espacios del Gabinete y la interacción con sus especialistas y dirección han sido posibles en gran parte gracias a la colaboración de la Coordinadora cubana Virgen Speck y de la facilitadora Yaquelín Jiménez, que por sus relaciones de trabajo ha contribuido a la presencia del proyecto en diferentes espacios. Con independencia de lo anterior, esta participación ha sido en la mayoría de las ocasiones resultado de nuestra gestión. En este sentido, se han producido reuniones de trabajo con el director del Gabinete, con miembros del Gabinete que atienden diferentes áreas de trabajo a los que se les presentó el Diagnóstico de Necesidades de Capacitación del Distrito y con el equipo de profesores de la Misión Académica del Gabinete.

También a partir de las relaciones de trabajo de la Coordinadora del Estado con actores sociales que se relacionan con el trabajo de la Misión hemos intervenido en actividades variadas como la Mesa de Intermisiones del Distrito Capital y un taller sobre implementación de la Ley de las Comunas. En el caso de esta última, dos profesores nuestros participaron en un Taller sobre la implementación de la Ley de las Comunas y urbanizaciones a los facilitadores que desarrollan sus acciones en este entorno, en calidad de oyentes (Ministerio de las Comunas), 16 de julio 2013.

Con independencia de la situación, se hace necesario destacar que todas las acciones de formación e investigación, como metodológicas durante los dos años fueron consultadas e informadas a la dirección del Gabinete cultural, en ocasiones por el Coordinador del equipo y en otras por la Coordinadora del estado y en todos los casos se manifestó por su dirección la satisfacción con el trabajo realizado.

\section{Reuniones de trabajo con equipo de facilitadores venezolanos para la concertación de}

\section{plan de formación.}

El equipo de facilitadores de la Misión Académica fue identificado por nuestro colectivo con motivo de las reuniones con la dirección del Gabinete (junio-julio 2013). A partir de la presentación del diagnóstico de necesidades de capacitación a los 17 facilitadores venezolanos de la Misión académica, se estableció un espacio de intercambio los lunes en la tarde, que ha solidificado nuestros lazos al coincidir estos profesores con los nuestros en varios puntos de vista respecto al enfoque metodológico del trabajo. Se han intercambiado temas sobre trabajo comunitario, andragogía, propuesta formativa de la misión académica, y retos y perspectivas del trabajo comunitario en urbanizaciones. Estos intercambios han permitido que ambos grupos hicieran patente la necesidad de realizar proyectos de abordaje de conjunto, en aras de favorecer la transformación sociocultural de las comunidades de la ciudad, conforme a los principios del Programa de la Patria, sin embargo dichas acciones no tuvieron todos los efectos deseados por diversas circunstancias propias de los facilitadores como: la reorganización del trabajo de la 
Misión académica en varias ocasiones, de la Misión Cultura en la que está inmersa también la Misión Cultura Corazón Adentro y las decisiones del equipo académico venezolano de esperar por circunstancias internas. No obstante, continuaron los espacios de intercambio los lunes en la tarde en la Fundación y se materializó hacia el trimestre enero-febrero-marzo 2014 la conformación de la Cátedra Permanente en el distrito capital y el desarrollo de diversas acciones formativas de conjunto entre facilitadores venezolanos y profesores cubanos dirigidas fundamentalmente a los animadores culturales en su primera etapa.

Durante el período de 2014 a 2015 el proceso de actualización del diagnóstico se realizó teniendo en cuenta los contenidos temáticos y ejes conceptuales establecidos para la actividad docente de la Cátedra Permanente "Soberanía y Cultura Comunal".

De conjunto con los facilitadores de la Misión Académica, la participación en este nuevo escenario permitió al proceso "Formación de Formadores" la revisión de los documentos normativos y metodológicos que reflejaban una actualización de las prioridades formativas del gabinete y la Fundación "Misión Cultura" en general.

Durante este período fue decisiva la integración con los facilitadores venezolanos puesto que representaron un amplio espectro de posibilidades con la Fundación Misión Cultura y el Gabinete, al mismo tiempo que permitieron, -desde cada uno de los encuentros de trabajo, discusión de documentos, proyección de estrategias y evaluación de resultados- la identificación de necesidades de capacitación y la correspondiente ejecución de las acciones formativas.

En todos los casos las propuestas temáticas planteadas reflejan aquellos contenidos en los que el equipo de profesores del proceso "Formación de Formadores", en articulación con los facilitadores de la misión académica, puede incidir desde sus ámbitos de actuación y competencias docentemetodológicas.

Esta labor ha sido fortalecida y continuada hasta la actualidad. De igual forma se han desarrollado talleres formativos entre los dos equipos de trabajo y la participación en actividades culturales y recreativas desarrollando los lazos de hermandad entre ambos.

\section{Articulación con diferentes actores y organizaciones sociales implicadas en las}

\section{estrategias de desarrollo cultural del Distrito Capital Libertador.}

Nuestro equipo durante los dos años de labor ha ido articulando con una serie de instituciones que participan en el desarrollo cultural del distrito, de forma directa o indirectamente. Esta articulación se ha desarrollado de forma autogestionada en la mayoría de los casos, por el nivel de socialización con actores sociales venezolanos que han sido beneficiarios de nuestras acciones, o han sido compañeros de labor en la facilitación de las mismas. En ocasiones, también por indicaciones de la coordinación nacional del proceso y la dirección del estado, de responder a la solicitud de un determinado organismo o actor. La participación del Gabinete Cultural del distrito y su dirección en el proceso de articulación con otros actores sociales e instituciones culturales a efectos de nuestra labor docente ha sido exigua y se ha limitado a su conocimiento y aprobación. 
De haber sido diferente, consideramos que el logro de los resultados hubiese sido superior.

Principales actores y organizaciones sociales con los que hemos articulado durante el transcurso de los dos años de trabajo:

- Sistema de Misiones, tanto con la parte venezolana como con la colaboración cubana.

- Centro de Investigación en Educación Popular (CIEP)

- Procesos esenciales de la Misión Cultura "Corazón Adentro" Formación Comunitaria y Educación por el Arte.

- Cátedra Permanente "Soberanía y Cultura Comunal"

- Museo de Bellas Artes,

- Casa de las Primeras Letras,

- Museo de Ciencias Naturales,

- Frente Francisco de Miranda,

- Escuela de Derechos Humanos de la Defensoría del Pueblo

- Subsecretaria de Educación del Distrito Capital,

- Universidad Bolivariana,

- Casa de Nuestra América José Martí,

- Escuela de Formación Carlos Miguel Escarra Malavé de la Asamblea Nacional

- Fundación "Oro Negro"

- Ministerio de las Comunas,

- Instituto de Juventudes,

- Instituto del Patrimonio Cultural.

- Madres del Barrio.

- Autoridad única Ciudad Caribia.

- Ministerio Público. 


\section{Conclusiones}

1. La etapa de inducción fue muy positiva, con excepción de la parte operativa y organizativa del proceso en Cuba (chequeo médico, llenado de planillas, el papel de D'ARTE en una comunicación transparente) y en Venezuela los aseguramientos logísticos en la etapa inicial. Respecto a la integración para el trabajo con otros proyectos esenciales de la misión, en el caso de Formación Comunitaria el proceso no tuvo la reciprocidad suficiente. En la segunda etapa, debido a las dinámicas impuestas por las políticas culturales del país en la atención las zonas de extrema pobreza y urbanismos se consolidó la integración de ambos procesos para el desarrollo de la labor docente y metodológica.

2. Durante todo el período, la organización y gestión de las acciones académicas fue desarrollada desde el equipo de "Formación de Formadores" y fueron identificadas las necesidades en un momento inicial por la colaboración cubana, luego se incorporaron paulatinamente las estructuras y actores sociales venezolanos. La periodicidad con que se han ejecutado las acciones docentes a los beneficiarios meta, fue lenta y espaciada en el tiempo durante el primer año, aunque se cumplió con los indicadores estadísticos del estado y con calidad y eficiencia en su desarrollo. En el segundo año el saldo cualitativo y cuantitativo fue superior pues además de los animadores culturales como beneficiarios meta y de especialistas pertenecientes a instituciones culturales se desarrolló la labor docente en las comunidades de extrema pobreza y nuevos urbanismos que incluyó sus pobladores y los miembros de sus diferentes estructuras de Poder Popular.

3. La labor investigativa ha sido desarrollada a partir de las propias acciones docentes, así como de dos grandes investigaciones: el diseño-actualización del diagnóstico y el proceso de sistematización de la experiencia. La experiencia del equipo de formación ha sido compartida con los profesores que han ingresado a la misión y transitado por nuestro equipo hacia nuevos estados o han pasado a la conformación de este.

4. Los procesos de coordinación y dirección del trabajo han sido colegiados por el grupo por lo general de manera colectiva. El cumplimiento de la planificación fue afectado al inicio por la dinámica propia del país y la imbricación que en este sentido tiene la colaboración cubana. En el segundo año esto fue superado lográndose mayor estabilidad. El equipo de profesores ha participado en diferentes espacios de trabajo a realizar con la Dirección del Gabinete, favorecidos por la gestión propia y por la coordinadora cubana de la Misión en el Distrito. Aún es insuficiente el reconocimiento de la importancia del proceso por los operadores y las diferentes plataformas a excepción del equipo de facilitadores de la misión académica. Estos profesores han sentido la necesidad de trabajar de conjunto con los nuestros en las acciones conjuntas de la Cátedra Permanente.

5. Se ha logrado la articulación con diversas instituciones y actores sociales que participan de forma directa o indirecta en el desarrollo cultural pero fundamentalmente por la autogestión de cada uno de los miembros del equipo. Se adolece de una estrategia de trabajo en ese orden por parte de la dirección del gabinete de caras al proceso y a las necesidades formativas de 
los diferentes actores sociales que contribuyen a la transformación sociocultural. El Proceso Formación de Formadores ha constituido desde su incorporación al Distrito un grupo asesor de la Dirección en cada uno de los procesos desde el diseño de la proyección estratégica, la propuesta de estructura y el sistema de control interno hasta su participación en el Consejo de Dirección y los principales órganos de dirección colegiada. De igual forma los profesores han desarrollado acciones docentes para cuadros y reservas. El colectivo se caracteriza por su calidad humana, lo que ha producido resultados positivos en el ámbito laboral y personal, sobre todo en la convivencia.

\section{Recomendaciones}

1. Fortalecer la articulación con los actores y funcionarios venezolanos, sus instituciones culturales y administrativas, así como otras que desde su misión contribuyen a la cultura (Gabinete de la fundación Misión Cultura, Concejos Comunales, cultores, líderes comunitarios, red de instituciones culturales, educativas, etc.).

2. Continuar el desarrollo de los procesos de autopreparación y estudio permanente en el equipo de profesores en beneficio de la calidad de las acciones docentes

3. Aumentar los niveles de gestión que aseguren una mayor disponibilidad de recursos didácticos, logísticos (computadora y materiales etc.), estabilidad y acceso a inmuebles pertinentes, para la preparación de los profesores y la impartición de las acciones académicas.

4. Se hace necesario diseñar y organizar, por parte de la dirección del Gabinete y los procesos formativos de la misión Cultura, una estrategia de integración y de acciones formativas, entre los operadores de las diferentes plataformas, los animadores, cultores y colaboradores cubanos, así como todas aquellas instituciones culturales o no, que tributen al desarrollo y la transformación sociocultural de las comunidades y actores sociales del distrito.

5. Continuar el trabajo conjunto e integrado de Formación Comunitaria y Formación de Formadores con vistas a perfeccionar el abordaje de las principales acciones formativas y de contribuir con mayor eficacia y eficiencia al desarrollo de su labor, con énfasis en zonas de extrema pobreza y urbanismos.

\section{Referencias Bibliográficas}

ALOBAN, HEGOA y Universidad de DEUSTO. La sistematización, una nueva mirada a nuestras prácticas. Guía para la sistematización de experiencias de transformación social. España. 2004.

ALONSO TEJEDA, María Eréndira, Cuadernos de formación de profesores № 3, Teorías del aprendizaje y la planeación didáctica, editado por Escuela Nacional Preparatoria, México, 2010.

ALVAREZ DE ZAYAS, C. (1989). Fundamentos teóricos de la dirección del proceso

ALVAREZ DE ZAYAS, C. (1994). Perfeccionamiento de los planes de estudio de la 
ALVAREZ DE ZAYAS. C. (1999). Didáctica. Pueblo y Educación. Habana

ANGULO, F y BLANCO, N. (2004). Teoría y desarrollo del currículum. Morata.

ARNAZ, J. A. (1981). La planeación curricular. Trillas. México.

BARBOSA CHACÓN, Jorge Winston; BARBOSA HERRERA, Juan Carlos y RODRíGUEZ VILLABONA, Margarita; "Revisión y análisis documental para estado del arte: una propuesta metodológica desde el contexto de la sistematización de experiencias educativas" en Investigación Bibliotecológica vol 27, N.61, UNAM Ciudad Universitaria, México, 2013.

BRUNER, J. (1988). Desarrollo Cognitivo y Educación. Madrid: Morata.

CAPÓ, W., Arteaga, B., Capó, M. y otros, La Sistematización de Experiencias: un método para impulsar procesos emancipadores, Fundación Editorial El perro y la rana, Caracas, Venezuela, 2010.

CARVAJAL BURBANO, Arizaldo (2010). Teoría y práctica de la sistematización de experiencias. 4ª . Edición, Cali, Escuela de Trabajo Social y Desarrollo Humano-Universidad del Valle.

CARVAJAL BURBANO, Arizaldo, Elementos de investigacion social aplicada Volumen 1 de Cuadernos de Cooperacion para el Desarrollo,Universidad del Valle, 2006.

CENDALES G. Lola y TORRES Alfonso "La sistematización como experiencia investigativa y formativa" en Revista GloobalHoy Ed. Consejo de Educación Popular de América Latina y el Caribe, España, 2003.

Colectivo de Autores, Selección de lecturas sobre Metodología de la Educación popular, CIE "Graciela Bustillos" Asociación de Pedagogos de Cuba, segunda edición, Ciudad de La Habana, 2001.

COLL, C. (1987). Psicología y Curriculum. LAIA. Barcelona. España.

CORRAL, R. (2002). Teoría y diseño curricular: Una propuesta desde el enfoque

DE ALBA, A. (19991). Evaluación Curricular. Conformación conceptual del campo.

“Diagnóstico Participativo, Mesa de Cultura y Plan de Acción Cultural”, Documento metodológico para el desarrollo de la Cátedra Permanente. Fundación Misión Cultura, Caracas, Venezuela, 2014

DÍAZ BARRIGA, F. (2000). Metodología de Diseño curricular para Educación.

DÍAZ, BARRIGA, A. (1980). Un enfoque metodológico para la elaboración de

DÍAZ, BARRIGA, A. (2000). Didáctica y Currículo. Edición Paidós. México 
Documento metodológico para el desarrollo de la Cátedra Permanente. Fundación Misión Cultura, Caracas, Venezuela, 2014.

“Educación Popular y la dimensión sociocultural del desarrollo”, en Grupos dinámicos de información, No. 8. Centro de Intercambio y referencia Iniciativa Comunitaria (CIERIC), 2010.

"Importancia de la planificación en el plan educativo", ed. Red Venezolana, 2014. El hogar de los venezolanos en la Red, en http://portal.redvenezolana.net/foros/importancia-de-la-planificacion-en-el-plan-educativo

GAGNER, R. Y BRIGGS, L. (2001). La planificación de la enseñanza. Trillas. México.

GUTIÉRREZ RICO, Dolores "Hablemos de Sistematización de Experiencias" en Revista Investigación Educativa Duraguense No. 8 de enero de 2008.

JARA H, Oscar Dilemas y desafíos de la sistematización de experiencias. Centro de Estudios y Publicaciones ALFORJA, Costa Rica

JARA H, Oscar, Para sistematizar experiencias. Una propuesta teórico-práctica. editorial, Alforja, 1994.

JARA H, Oscar, Trayectos de la sistematización de experiencias en América Latina 1959-2010, Centro de Estudios y Publicaciones Alforja, 2010.

JARA, H, Oscar, La Sistematización de Experiencias: Práctica y Teoría para otros mundos posibles, $2 d a$ Ed. Fundación Centro Internacional de Educación y Desarrollo Humano CINDE, Colombia, 2019.

JARA, H, Oscar. Dilemas y desafíos de la sistematización de experiencia. Centro de estudios y publicaciones Alforja. Costa Rica. 1999.

La Sistematización como creación de saber de liberación. Colaborador Asociación de Instituciones de Promoción y Educación (La Paz, Bolivia), ed, AIPE, 1987.

La Sistematización como práctica: cinco experiencias con sectores populares Volumen $\quad 6 \quad$ de Nuevos cuadernos Volumen 6 de Nuevo cuaderno: Centro Latinoamericano de Trabajo Social Colaborador Centro Latinoamericano de Trabajo Social, editor: Centro Latinoamericano de Trabajo Social, 1985.

Laurencio Barraza (Coordinador General); Diagnóstico sobre la realidad social, económica y cultural de los entornos locales para el diseño de intervenciones en materia de prevención y erradicación de la violencia en la región norte: el caso de ciudad juárez, chihuahua; Ed. Comisión Nacional para Prevenir y Erradicar la Violencia contra las mujeres, México 2009.

Leonel Sánchez; Diagnóstico social participativo; Ed. Fundación Escuela Gerencia Social, Ministerio de Planificación y Desarrollo, PDVSA, Caracas, Venezuela, 2005. 
LEWIN, K. "La investigación-acción y los problemas de las minorías". En AA.VV., La investigación- acción participativa. Inicio y desarrollo, Biblioteca de Educación de Adultos, Nº 6, Edición. Popular, Madrid, 1992.

MIDDELTON David y EDWARDS, Derek, Memoria Compartida: la naturaleza social del recuerdo y del olvido; ed. Paidos Barcelona, 1992.

MORENO GAJARDO, Florelina Angélica; "Planificación docente", Red Maestros de Maestros, 2014, en http:// www.rmm.cl/index sub.php?id contenido=15510\&id seccion=2271\&id portal=354

MORGAN, María de La Luz. Taller Permanente de Sistematización. Búsquedas Teóricas y Epistemológicas desde la Practica de la Sistematización. Lima. 1996.

Planeación didáctica. Evidencias de la práctica docente, ed. Universidad de Guanajuato. División de Ciencias Naturales y Exactas, 2012.

Planeación didáctica. Evidencias de la práctica docente, ed. Universidad de Guanajuato. División de Ciencias Naturales y Exactas, 2012.

"Planificación de la educación", ed. Ministerio del Poder Popular para la Educación Universitaria, Venezuela, 2014, en http://www.bibliodar.mppeu.gob.ve/?q=doc categoria/Planificaci\%C3\%B3n\%20de\%20la\%20 educaci\%C3\%B3n

"Planificación de la educación", ed. Ministerio del Poder Popular para la Educación Universitaria, Venezuela, 2014, en http://www.bibliodar.mppeu.gob.ve/?q=doc categoria/Planificaci\%C3\%B3n\%20de\%20la\%20 educaci\%C3\%B3n

Programas docentes de la Cátedra Permanente "Soberanía y Cultura Comunal" CD, Ministerio del Poder Popular para la Cultura, Caracas, Venezuela, 2013

RODRIGUEZ SOSA, Jorge, "Sistematización de una experiencia de capacitación de docentes en servicio mediante el empleo de la investigación-acción" en Propósitos y Representaciones vol 7, No. 1, Universidad San Ignacio de Loyola, Lima, Perú, 2019.

SANCHO, J.M. (2001). La problemática de la evaluación. LAIA. Barcelona

SANIN BETANCOURT, Maria Clara, Guía Orientadora para la Sistematización de Experiencias. Objetivos de Desarrollo Sostenible y Cooperación Sur-Sur. Serie de Documentos de Trabajo. Ed. Secretaría General Iberoamericana- Programa Iberoamericano para el Fortalecimiento de la Cooperación Sur-Sur, San Salvador, 2016.

STEHOUSE, L. (2007). Investigación y desarrollo del currículum. Morata. Madrid.

Torrealba , Marielys y Bethzabe Piña Naudy Soto, "El docente novel y la planificación pedagógico administrati- 
va del proceso enseñanza aprendizaje en educación primaria”, 2014, ed. Instituto Pedagógico de Braquisimeto, Venezuela, en: http://revistas.upel.edu.ve/index.php/educare/article/view/359

VIÑAS PÉREZ, Gladys, Métodos y Técnicas participativas en el proceso de enseñanza aprendizaje, Ed, CEPES Universidad de La Habana, 2010.

Yolanda Molina, Omar Carrero Gámez, Omar Carrero Araque, Alberto Villarreal, Ernesto Arends, Juan Santaromita, Herlinda Coronado, Francisco Sánchez y Domingo Sánchez; El diagnóstico participativo para el desarrollo integral comunitario en el marco de la Ley de los Consejos Comunales: Un caso práctico en comunidades Piaroa del estado Amazonas, Ed. Revista Forestal Latinoamericana, 23(2):77-109. 2008

ZABALZA BERAZA, Miguel A., "Guia para la planificación didáctica en la docencia Universitaria en el marco del EES (Guía de guías)", documento de trabajo, ed. Universidad Santiago de Compostela, octubre 2004, en https://www.uclm.es/profesorado/ricardo/Convergencia/guiaplan aZABALZA.pdf 
